\title{
Clinical effectiveness of treatment with hyperbaric oxygen for neonatal hypoxic-ischaemic encephalopathy: systematic review of Chinese literature
}

Zulian Liu, Tengbin Xiong, Catherine Meads

\begin{abstract}
Objectives To investigate the clinical effectiveness of treatment with hyperbaric oxygen for neonates with hypoxic-ischaemic encephalopathy. This treatment is frequently used in China but much less often in the West.

Data sources Western (Cochrane controlled trials register and database of systematic reviews, Medline, Embase, CINAHL, and HealthSTAR) and Chinese (China Hospital Digital Library, Chinese Medical Journal Network) databases and hand search of Chinese journals. No language restrictions.

Review methods Randomised or quasi-randomised controlled trials of treatment with hyperbaric oxygen compared with "usual care" in term neonates with hypoxic-ischaemic encephalopathy. Outcomes included mortality and long term neurological sequelae. Standardised forms were used to extract and compare data. Criteria of York Centre for Reviews and Dissemination were used to assess quality. Analysis was mainly qualitative but included meta-analysis.

Results 20 trials were found, mainly from Chinese sources. The reporting quality of trials was poor by Western (CONSORT) standards. Treatment with hyperbaric oxygen had better outcomes than the comparator in almost all trials. The odds ratios of the meta-analyses were 0.26 (95\% confidence interval 0.14 to 0.46 ) for mortality and $0.41(0.27$ to 0.61$)$ for neurological sequelae.

Conclusion Treatment with hyperbaric oxygen possibly reduces mortality and neurological sequelae in term neonates with hypoxic-ischaemic encephalopathy. Because of the poor quality of reporting in all trials and the possibility of publication bias, an adequately powered, high quality randomised controlled trial is needed to investigate these findings. The Chinese medical literature may be a rich source of evidence to inform clinical practice and other systematic reviews.
\end{abstract}

\section{Introduction}

Hypoxic-ischaemic encephalopathy is a severe complication of asphyxia that occurs before, during, or after birth. It can result in death or neurological damage, which can manifest in the short term (within 12-24 hours) as seizures, altered reflexes, or altered level of consciousness (or a combination), and in the longer term by developmental delay, epilepsy, mental retardation, or cerebral palsy (or a combination). Diagnosis is by a history of asphyxia that has caused acidaemia, a low Apgar score, neurological damage, and the involvement of many organs. The condition is commonly graded as mild, moderate, or severe. Sarnat stages can be used to classify the neurological damage-stage I is least severe and stage III most severe. ${ }^{1}$ The condition occurs in 3.5-6/1000 live births, and the outcome is worse for more severely affected neonates. ${ }^{2}$ One case series of 38 births reported 14 deaths and 13 patients with a poor outcome. ${ }^{3}$ In another series of 42 survivors with moderate hypoxic-ischaemic encephalopathy followed up at one year, two were dead, 13 had cerebral palsy, one had another severe disability, four had mild developmental delay, and 22 had developed normally. ${ }^{1}$ Treatments evaluated for this condition include hypothermia, magnesium sulphate, anticonvulsants, mannitol, dexamethasone, nicardipine, and caffeic acid phenethyl ester, but none has been effective. ${ }^{4-7}$ Management usually consists of supportive care and keeping oxygen saturation at $95 \%{ }^{2}$

Patients treated with hyperbaric oxygen inhale $100 \%$ oxygen inside a hyperbaric chamber that is pressurised to $>0.1 \mathrm{MPa}$ (megapascals). This treatment has been evaluated in the West for a wide range of conditions, including cerebral oedema, brain injuries, and cerebral palsy, but not for hypoxic-ischaemic encephalopathy. ${ }^{8-10}$ In Russia, hyperbaric oxygen has been used to treat neonatal injuries (fetal asphyxia), and this treatment is used for hypoxic-ischaemic encephalopathy in China, but apparently not in Hong Kong. ${ }^{11-13}$ Hyperbaric oxygen is usually given one to three times per day at $0.15-0.17 \mathrm{MPa}$ for $60-120$ minutes, with the aim of increasing oxygen in the tissues. ${ }^{14}$ The rationale for this treatment is that it may reverse local hypoxia, inhibit post-ischaemic vasoconstriction, and promote the formation of collagen matrix, which is essential for angiogenesis and restoration of blood flow to injured tissue. ${ }^{14}$ This systematic review investigates whether hyperbaric oxygen is clinically effective for the treatment of neonates born at term with hypoxic-ischaemic encephalopathy.

\section{Methods}

The protocol for this systematic review was developed as part of a masters degree in health technology assessment.

The search strategy comprised a search of Western electronic databases and a search of Chinese databases and other sources. We searched the Cochrane controlled trials register and database of systematic reviews, Medline, Embase, CINAHL, and HealthSTAR up to November 2004 using search terms "hyperbaric oxygen", "hyperbaric oxygenation", "neonate(s)", "newborn(s)", "infant newborn(s)", "hypoxic-ischemic", "encephalopathy", "encephalopathies", "brain injury", "brain injuries", "brain damage", "brain ischemia", "hypoxia brain", and "birth asphyxia". We also searched a variety of Chinese electronic 
Table 1 Characteristics of 20 trials investigating treatment with hyperbaric oxygen in hypoxic-ischaemic encephalopathy

\begin{tabular}{|c|c|c|c|c|c|c|c|c|}
\hline \multirow[t]{2}{*}{ Study } & \multicolumn{2}{|c|}{ No of patients* } & \multirow[t]{2}{*}{$\begin{array}{l}\text { Diagnostic criteria } \\
\left(\text { severity }^{*}\right)\end{array}$} & \multirow[t]{2}{*}{ Baseline comparisons } & \multicolumn{2}{|c|}{ Age at start of treatment } & \multirow[t]{2}{*}{ Treatment‡ } & \multirow[t]{2}{*}{ Usual care } \\
\hline & Intervention & Control & & & Treatment & Usual care† & & \\
\hline Chen $2000^{17}$ & 37 & 36 & $\begin{array}{l}\text { Ji Nan conference } \\
\text { (I, II, and III) }\end{array}$ & $\begin{array}{l}\text { No statistically significant } \\
\text { difference in status of } \\
\text { consciousness, convulsion, } \\
\text { muscle tone, reflexes, or } \\
\text { computed tomography } \\
\text { results }\end{array}$ & $2-5$ days & NA & $\begin{array}{l}0.14-0.16 \mathrm{MPa} \\
\text { (concentration=80\%); } 20 \mathrm{~min} / 30 \\
\text { min/20 min; once a day } \times 10 \text { days; } \\
1-5 \text { courses; not clear }\end{array}$ & General \\
\hline Ding $2003^{18}$ & 92 & 86 & $\begin{array}{l}\text { Han Zhou } \\
\text { conference (NA) }\end{array}$ & $\begin{array}{l}\text { No statistically significant } \\
\text { difference in sex, age, status } \\
\text { of asphyxia, or clinical } \\
\text { symptoms }\end{array}$ & $<48$ hours & $<24$ hours & $\begin{array}{l}0.15-0.17 \mathrm{MPa} ; 15 \mathrm{~min} / 30 \mathrm{~min} / 20 \\
\text { min; once a day } \times 10 \text { days; not } \\
\text { clear; not clear }\end{array}$ & $\begin{array}{l}\text { General and } \\
\text { drug that } \\
\text { promotes } \\
\text { cerebral } \\
\text { metabolism }\end{array}$ \\
\hline He $2000^{19}$ & $\begin{array}{l}\text { I: } 4 ; \text { II: } 18 \\
\text { III: } 10\end{array}$ & $\begin{array}{l}\text { I: } 4 ; \text { II: } 17 \\
\text { III: } 9\end{array}$ & $\begin{array}{l}\text { Han Zhou } \\
\text { conference (I, II, } \\
\text { and III) }\end{array}$ & $\begin{array}{l}\text { No statistically significant } \\
\text { difference in birth weight, } \\
\text { degree of asphyxia, or main } \\
\text { clinical symptoms }\end{array}$ & $<72$ hours & $<24$ hours & $\begin{array}{l}0.15 \mathrm{MPa} ; 15 \mathrm{~min} / 30 \mathrm{~min} / 15 \text { to } 30 \\
\text { min; once a day } \times 10 \text { days; not } \\
\text { clear; not clear }\end{array}$ & $\begin{array}{l}\text { General and } \\
\text { drug that } \\
\text { promotes } \\
\text { cerebral } \\
\text { metabolism }\end{array}$ \\
\hline Li $2004^{20}$ & 20 & 20 & $\begin{array}{l}\text { Hu and Jiang (I, II, } \\
\text { and III) }\end{array}$ & $\begin{array}{l}\text { No statistically significant } \\
\text { difference in birth weight, } \\
\text { sex, age, or Apgar score }\end{array}$ & $\begin{array}{l}\text { I and II: } 15 \\
\text { hours to } 7 \text { days; } \\
\text { III: }>7 \text { days }\end{array}$ & NA & $\begin{array}{l}\text { Not clear; not clear; } 10 \text { times; I and } \\
\|=1-2 \text { courses, III=3-5 courses; } \\
10-15 \text { days }\end{array}$ & General \\
\hline Lin $2000^{21}$ & 30 & 30 & NA (I, II, and III) & $\begin{array}{l}\text { No statistically significant } \\
\text { difference in ways of delivery, } \\
\text { sex, gestational age, birth } \\
\text { weight, age, or clinical grade }\end{array}$ & NA & $\leq 1$ day & $\begin{array}{l}\text { Not clear; not clear; once a day } \times 10 \\
\text { days; not clear; not clear }\end{array}$ & General \\
\hline Liu $2003^{22} \S$ & II: $61 ;$ III: 39 & II: $60 ;$ III: 38 & $\begin{array}{l}\text { Han Zhou } \\
\text { conference (II and } \\
\text { III) }\end{array}$ & $\begin{array}{l}\text { No statistically significant } \\
\text { difference in sex, birth } \\
\text { weight, illness status, } \\
\text { maternity age, parents' social } \\
\text { status, or family status }\end{array}$ & Within 24 days & $\begin{array}{l}\text { Within } 24 \\
\text { days }\end{array}$ & $\begin{array}{l}0.15-0.17 \mathrm{MPa} ; 15 \mathrm{~min} / 30 \mathrm{~min} / 20 \\
\text { min; once a day } \times 10 \text { days; not } \\
\text { clear; not clear }\end{array}$ & General \\
\hline Lu $2001^{23}$ & II: 19 ; III: 10 & II: 16; III: 8 & $\begin{array}{l}\text { Han Zhou } \\
\text { conference (II and } \\
\text { III; excluded } \\
\text { cerebral } \\
\text { haemorrhage) } \\
\end{array}$ & $\begin{array}{l}\text { No statistically significant } \\
\text { difference in birth weight, } \\
\text { gestational age, time starting } \\
\text { treatment, or clinical grade }\end{array}$ & $2-8$ days & NA & $\begin{array}{l}0.12 \mathrm{MPa} .20 \mathrm{~min} / 30 \mathrm{~min} / 20 \mathrm{~min} \text {; } \\
\text { once; not clear; not clear }\end{array}$ & General \\
\hline Lu $2003^{24}$ & 37 & 34 & $\begin{array}{l}\text { Han Zhou } \\
\text { conference (II and } \\
\text { III; included } \\
\text { cerebral } \\
\text { haemorrhage) } \\
\end{array}$ & $\begin{array}{l}\text { No statistically significant } \\
\text { difference in sex, birth } \\
\text { weight, or degree of asphyxia } \\
\text { and anoxia }\end{array}$ & $\begin{array}{l}<48 \text { hours after } \\
\text { admission; } \\
\text { stable if with } \\
\text { cerebral } \\
\text { haemorrhage }\end{array}$ & NA & $\begin{array}{l}0.16 \mathrm{MPa} ; 20 \mathrm{~min} / 30 \mathrm{~min} / 20 \mathrm{~min} \\
\text { once a day } \times 10 \text { days; } \mathrm{II}=2 \text { courses, } \\
\mathrm{II}=3 \text { courses; not clear }\end{array}$ & $\begin{array}{l}\text { General and } \\
\text { drugs that } \\
\text { promote } \\
\text { cerebral } \\
\text { metabolism }\end{array}$ \\
\hline Lu $1999^{25} \S$ & $\begin{array}{l}\text { I: } 15 ; \text { II: } 11 \\
\text { II: } 6\end{array}$ & $\begin{array}{l}\text { I: } 12 ; \text { II: } 13 ; \\
\text { III: } 5\end{array}$ & $\begin{array}{l}\text { Han Zhou } \\
\text { conference (I, II, } \\
\text { and III) }\end{array}$ & $\begin{array}{l}\text { No statistically significant } \\
\text { difference in sex, birth } \\
\text { weight, status of asphyxia } \\
\text { and anoxia, or clinical } \\
\text { symptoms }\end{array}$ & $<48$ hours & $<24$ hours & $\begin{array}{l}0.15-0.17 \mathrm{MPa} ; 15 \mathrm{~min} / 30 \mathrm{~min} / 20 \\
\text { min; once a day } \times 10 \text { days; not } \\
\text { clear; not clear }\end{array}$ & $\begin{array}{l}\text { General and } \\
\text { drugs that } \\
\text { promote } \\
\text { cerebral } \\
\text { metabolism and } \\
\text { eliminate free } \\
\text { radicals }\end{array}$ \\
\hline Si $1999^{26}$ & $\begin{array}{l}\text { I: } 11 ; \text { II: } 27 \\
\text { III: } 19\end{array}$ & $\begin{array}{l}\text { I: } 10 ; \text { II: } 22: \\
\text { III: } 17\end{array}$ & $\begin{array}{l}\text { Han Zhou } \\
\text { conference (I, II, } \\
\text { and III; excluded } \\
\text { those with brain } \\
\text { malformation and } \\
\text { severe } \\
\text { decompensation) }\end{array}$ & $\begin{array}{l}\text { No statistically significant } \\
\text { difference in sex, birth weight } \\
\text { and status of asphyxia. }\end{array}$ & $\begin{array}{l}<48 \text { hours after } \\
\text { admission }\end{array}$ & NA & $\begin{array}{l}0.2 \mathrm{MPa} ; 20 \mathrm{~min} / 60 \mathrm{~min} / 20 \mathrm{~min} ; \\
\text { once a day } \times 7 \text { days; not clear; not } \\
\text { clear }\end{array}$ & General \\
\hline Song $2000^{27} \S$ & 51 & 50 & $\begin{array}{l}\text { Han Zhou } \\
\text { conference (II and } \\
\text { III) }\end{array}$ & $\begin{array}{l}\text { No statistically significant } \\
\text { difference in sex, birth } \\
\text { weight, clinical grade, } \\
\text { maternity age, and family } \\
\text { status }\end{array}$ & $<48$ hours & $<24$ hours & $\begin{array}{l}0.15-0.17 \mathrm{MPa} ; 15 \mathrm{~min} / 30 \mathrm{~min} / 20 \\
\text { min; once a day } \times 10 \text { days; not } \\
\text { clear; not clear }\end{array}$ & General \\
\hline Sun $2000^{28}$ & I: $21 ;$ III: 21 & I: 19; III: 21 & $\begin{array}{l}\text { Ji Nan conference } \\
\text { (I and III) }\end{array}$ & NA & NA & $<3$ days & NA & $\begin{array}{l}\text { General (3rd } \\
\text { arm not } \\
\text { included) }\end{array}$ \\
\hline Sun $1998^{29}$ & $\begin{array}{l}\text { I: } 10 ; \text { II: } 18 \\
\text { III: } 4\end{array}$ & $\begin{array}{l}\text { I: } 7 ; \text { II: } 13 ; \\
\text { III: } 2\end{array}$ & $\begin{array}{l}\text { Ji Nan conference } \\
\text { (I, II, and III; } \\
\text { included } \\
\text { complications of } \\
\text { cerebral } \\
\text { haemorrhage) }\end{array}$ & $\begin{array}{l}\text { No statistically significant } \\
\text { difference in various aspects } \\
\text { including symptoms }\end{array}$ & NA & NA & $\begin{array}{l}0.14 \mathrm{MPa}(1.4 \mathrm{~atm}) ; 15 \mathrm{~min} / 40 \\
\mathrm{~min} / 20 \mathrm{~min} \text {; once a day ×7-10 days; } \\
\text { not clear; not clear }\end{array}$ & General \\
\hline Wang $2002^{30}$ & $\begin{array}{l}\text { I: } 15 ; \text { II: 22; } \\
\text { III: } 11\end{array}$ & $\begin{array}{l}\text { I: } 16 ; \text { II: } 20 \\
\text { III: } 10\end{array}$ & $\begin{array}{l}\text { Ji Nan conference } \\
\text { (all) }\end{array}$ & $\begin{array}{l}\text { No statistically significant } \\
\text { difference in sex, birth } \\
\text { weight, or degree of asphyxia } \\
\text { and anoxia }\end{array}$ & NA & $<2-3$ days & $\begin{array}{l}0.14 \mathrm{MPa} ; 20 \mathrm{~min} / 50 \mathrm{~min} / 25 \mathrm{~min} ; 7 \\
\text { days; I=1 course, II=2-3 courses, } \\
\text { III=3-4 courses; } 3-5 \text { days }\end{array}$ & $\begin{array}{l}\text { General, } \\
\text { antioxidants, } \\
\text { and cerebrolysin }\end{array}$ \\
\hline Wang $1999^{31}$ & II: 14; III: 9 & II: 15; III: 7 & $\begin{array}{l}\text { Han Zhou } \\
\text { conference (II and } \\
\text { III; excluded } \\
\text { cerebral } \\
\text { haemorrhage from } \\
\text { birth injury and } \\
\text { malformation) } \\
\end{array}$ & $\begin{array}{l}\text { No statistically significant } \\
\text { difference in sex, birth } \\
\text { weight, gestational age, or } \\
\text { Apgar score }\end{array}$ & 72 hours & NA & $\begin{array}{l}0.15-0.16 \mathrm{MPa} ; \\
\text { (concentration=80\%); } 20 \mathrm{~min} / 30 \\
\text { min/20-30 min; once } \times 5-8 \text { days; } \\
\text { not clear; not clear }\end{array}$ & $\begin{array}{l}\text { General and } \\
\text { nicholin }\end{array}$ \\
\hline
\end{tabular}




\begin{tabular}{|c|c|c|c|c|c|c|c|c|}
\hline Wang $2001^{32}$ & 801 & $\begin{array}{l}\text { I: } 24 ; \text { II: } 36 ; \\
\text { III: } 20\end{array}$ & $\begin{array}{l}\text { Defined in text (I, } \\
\text { II, and III) }\end{array}$ & $\begin{array}{l}\text { No statistically significant } \\
\text { difference in illness status }\end{array}$ & $\begin{array}{l}\text { As early as } \\
\text { possible }\end{array}$ & NA & $\begin{array}{l}\text { Not clear; } 20 \mathrm{~min} / 60 \mathrm{~min} / 20 \mathrm{~min} \text {; } \\
\text { once a day } \times 10 \text { days; not clear; not } \\
\text { clear }\end{array}$ & General \\
\hline Wang $2001^{33}$ & $\begin{array}{l}\text { I: } 26 ; \text { II: 46; } \\
\text { III: } 12\end{array}$ & $\begin{array}{l}\text { I: } 24 ; \text { II: 45; } \\
\text { III: } 11\end{array}$ & $\begin{array}{l}\text { Jin and Huang (I, } \\
\text { II, and III; except } \\
\text { congenital disease) }\end{array}$ & $\begin{array}{l}\text { No significant difference in } \\
\text { age, gestational age, illness } \\
\text { status, or family status }\end{array}$ & NA & $\begin{array}{l}30 \text { min to } 3 \\
\text { days }\end{array}$ & $\begin{array}{l}0.15 \mathrm{MPa} \text {; total time }=60-70 \mathrm{~min} \\
\text { each time; once a day } \times 5-7 \text { days; } \\
\text { not clear; not clear }\end{array}$ & General \\
\hline Wen $2001^{34}$ & 38 & 38 & $\begin{array}{l}\text { Han Zhou } \\
\text { conference (II and } \\
\text { III) }\end{array}$ & $\begin{array}{l}\text { No significant difference in } \\
\text { sex or age }\end{array}$ & $<3$ days & NA & $\begin{array}{l}0.13 \mathrm{MPa} ; 15 \mathrm{~min} / 30 \mathrm{~min} / 15 \mathrm{~min} ; \\
\text { once a day } \times 10 \text { days; not clear; not } \\
\text { clear }\end{array}$ & General \\
\hline Yuan $1999^{35}$ & $\begin{array}{l}\text { I: } 16 ; \text { II: } 9 \text {; } \\
\text { III: } 5\end{array}$ & $\begin{array}{l}\text { I: } 17 ; \text { II: } 8 \text {; } \\
\text { III: } 5\end{array}$ & $\begin{array}{l}\text { Ji Nan conference } \\
\text { (I, II, and III) }\end{array}$ & $\begin{array}{l}\text { Listed symptoms but did not } \\
\text { compare }\end{array}$ & $\begin{array}{l}\text { Within } 2 \text { days of } \\
\text { admission }\end{array}$ & 24-48 hours & $\begin{array}{l}0.14-0.16 \mathrm{MPa} ; 20 \mathrm{~min} / 30 \\
\mathrm{~min} / 20-30 \mathrm{~min} ; \text { not clear; I=2-3 } \\
\text { times, II=5 times, III=10 times; not } \\
\text { clear }\end{array}$ & General \\
\hline Zhang $2000^{36}$ & 60 & 56 & $\begin{array}{l}\text { Jinan conference (II } \\
\text { and III) }\end{array}$ & $\begin{array}{l}\text { No statistically significant } \\
\text { difference in sex, gestational } \\
\text { age, birth weight, or Apgar } \\
\text { score }\end{array}$ & NA & NA & $\begin{array}{l}0.16 \mathrm{MPa}, 10-15 \mathrm{~min} / 30 \mathrm{~min}, / 15-20 \\
\text { min; once a day } \times 10 \text { days; II=2 } \\
\text { courses, III=3 courses; } 1 \text { week }\end{array}$ & General \\
\hline
\end{tabular}

$\mathrm{MPa}$, megapascals; NA=not available.

* Severity grade: I=mild, II=moderate, III=severe.

†The age starting usual care was assumed as the time of admission.

†Pressure; time of increasing/stable/decreasing pressure; 1 course; length; interval. Time is the duration increasing/stable/decreasing pressure of a single treatment, length is the number of courses of treatment, and interval is the time between two courses of treatment.

§Alternate patient allocation.

१The numbers given for severity grades I (26) II (37) and III (21) add up to 84 but the total number in the intervention group was given as 80 .

databases and hand searched selected Chinese journals (see box) to July 2004.

We identified relevant studies by searching electronic databases, scanning reference lists, and consulting experts in the specialty. Publications in any language were eligible. Reference lists were hand searched for further references. We examined titles, abstracts, and keywords of citations as given on the databases for the terms for "hyperbaric oxygen therapy for neonatal hypoxic-ischaemic encephalopathy". Where possible, we obtained the full text of all potentially relevant citations.

The predetermined inclusion criteria were fully published randomised or quasi-randomised controlled trials of treatment with hyperbaric oxygen compared with "usual care" in full term neonates (more than 36 weeks' gestation) with hypoxicischaemic encephalopathy and a history of perinatal asphyxia. We accepted alternate allocation as quasi-randomised. Outcomes were mortality and incidence of long term neurological sequelae (developmental delay, epilepsy, mental retardation, or cerebral palsy, or a combination). One reviewer (ZL) assessed studies for inclusion and this was checked independently by a second reviewer (TX). Both reviewers independently extracted data from the papers using a standardised, predesigned data

\section{Chinese data sources}

\section{Electronic databases}

China Hospital Digital Library, administered by the Department of Education of China and sponsored by Tsinghua University and the Chinese Medical Association. This database provides full text data from published periodicals and newspapers in China on medical specialties, bioscience, government of hospitals, library science, and information science. The main component is the China hospital knowledge database, which contains more than 1300 professional periodicals and 3000 related periodicals; it covers $96 \%$ of articles on medicine published in China. Relevant keywords were used for searching

An online bibliographical database from the China Hyperbaric Oxygen Medicine Information Centre, which contains data on relevant literature published in Chinese medical journals Chinese Medical Journal Network for full text medical literature

Hand searches

Selected journals in Chinese: Journal of Clinical Pediatrics and Chinese Journal of Practical Pediatrics extraction form, and no disagreements were encountered. We assessed the quality of the included trials by using criteria of the York Centre for Reviews and Dissemination; we focused on randomisation, allocation concealment, presence of blinding, explanation of withdrawals, and presence or absence of intention to treat analysis. ${ }^{15}$

We tabulated the characteristics and results of all the included studies; analysis was mainly qualitative. We carried out meta-analysis using Metaview 4.1 (Cochrane Collaboration Review Manager 4.1 software). We used fixed effects models when statistical heterogeneity was absent and random effects models when heterogeneity was present. Statistical heterogeneity was present if $\chi^{2}$ values were greater than the degrees of freedom. ${ }^{16}$

\section{Results}

We found six citations in Western databases, but none met the inclusion criteria. We identified 126 citations from the Chinese searches. Twenty trials met the inclusion criteria and 106 were excluded (59 had the wrong study design, 37 did not specify the term neonate, two had different interventions, and eight had different outcomes). ${ }^{17-36}$ All of the included trials were conducted in China and published in Chinese language medical journals. The

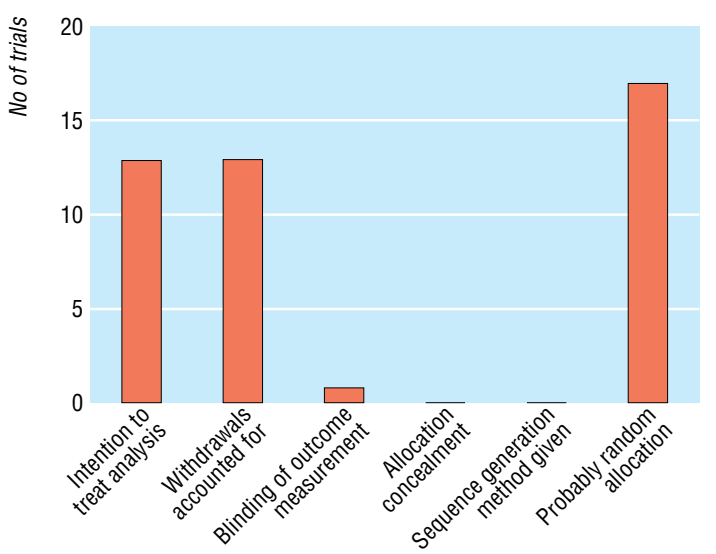

Fig 1 Quality assessment of trials of hyperbaric oxygen to treat hypoxic-ischaemic encephalopathy 


\begin{tabular}{|c|c|c|c|c|c|c|}
\hline Study & $\begin{array}{l}\text { Total effectiveness rate (measurement } \\
\text { index) }\end{array}$ & Mean (SD) days to recovery & $\begin{array}{l}\text { Length of follow-up; } \\
\text { losses to follow-up }\end{array}$ & $\begin{array}{l}\text { Long term neurological } \\
\text { sequelae }\end{array}$ & Mortality & $\begin{array}{l}\text { Adverse } \\
\text { events }\end{array}$ \\
\hline Chen $2000^{17}$ & NA & $\begin{array}{l}\text { Seizures controlled, consciousness, } \\
\text { and muscle tone. 2-5 fewer days in } \\
\text { I than C }\end{array}$ & 12-24 months; none & $\begin{array}{l}\text { I: } 8.1 \%(3 / 37), \text { C: } 19.4 \% \\
\text { (7/36) (numbers in text } \\
\text { do not add up) }\end{array}$ & $\begin{array}{l}\text { I: } 5.4 \%(2 / 37) \\
\text { C: } 5.5 \%(2 / 36)\end{array}$ & NA \\
\hline Ding $2003^{18}$ & $\begin{array}{l}\text { I did significantly better than } \mathrm{C} ; \mathrm{P}<0.005 \\
\text { (clinical symptoms disappeared in } 10 \text { days) }\end{array}$ & NA & $>10$ days; NA & NA & NA & None \\
\hline He $2000^{19}$ & $\begin{array}{l}\text { I: } 94 \%(30 / 32), \text { C: } 67 \%(20 / 30) ; P<0.01 \\
\text { (clinical symptoms disappeared in } 10 \text { days) }\end{array}$ & $\begin{array}{l}\text { Days in hospital. I: } 12.5 \text { (2.8), C: } \\
15.5 \text { (3.1) }\end{array}$ & NA; none & NA & $\begin{array}{l}\text { I: } 0 \%(0 / 32), \text { C: } \\
6.7 \%(2 / 30)\end{array}$ & None \\
\hline Li $2004^{20}$ & $\begin{array}{l}\text { I: } 90 \% \text { (18/20), C: } 60 \%(12 / 20) ; \mathrm{P}<0.05 \\
\text { (clinical symptoms-cerebral oedema by } \\
\text { ultrasound disappeared in } 5 \text { days) }\end{array}$ & NA & 3 years; probably none & $\begin{array}{l}\text { I: } 10.0 \%(2 / 20), \text { C: } \\
20.0 \%(4 / 20)\end{array}$ & NA & None \\
\hline Lin $2000^{21}$ & $\begin{array}{l}\text { I: } 97 \% \text { (29/30), C: } 73 \%(22 / 30) ; P<0.05 \\
\text { (clinical conditions improved in } 1 \text { week) }\end{array}$ & NA & $\begin{array}{l}5 \text { months to } 2 \text { years; } \\
\text { none for total } \\
\text { effectiveness rate, } 7 \text { for } \\
\text { neurological sequelae }\end{array}$ & $\begin{array}{l}\text { I: } 3.3 \%(1 / 30), \text { C: } 23.3 \% \\
(7 / 30)\end{array}$ & NA & NA \\
\hline Liu $2003^{22 *}$ & $\begin{array}{l}\text { I: } 90 \%(90 / 100), C: 67 \% \text { (66/98); } P<0.01 \\
\text { (clinical symptoms disappeared in } 10 \text { days) }\end{array}$ & NA & 6-14 months; 34 & $\begin{array}{l}\text { I: } 20.4 \%(18 / 100), \text { C: } \\
44.7 \%(34 / 98)\end{array}$ & $\begin{array}{l}\text { I: } 4.0 \%(4 / 100) \text {, } \\
\text { C: } 20.4 \% \\
(20 / 98) ; P<0.05\end{array}$ & NA \\
\hline Lu $2001^{23}$ & NA & $\begin{array}{l}\text { Symptoms. I: } 4.3 \text { (2.3), C: } 5.8 \text { (2.0); } \\
\mathrm{P}<0.001\end{array}$ & NA; none & NA & $\begin{array}{l}\text { I: } 0 \%(0 / 29), \text { C: } \\
4.2 \%(1 / 24)\end{array}$ & None \\
\hline Lu $2003^{24}$ & $\begin{array}{l}\text { I: } 92 \% \text { (34/37), C: } 74 \%(25 / 34) ; \text { P }<0.01 \\
\text { (clinical symptoms—cerebral } \\
\text { oedema-disappeared in } 10 \text { days; how } \\
\text { cerebral oedema was detected not given) }\end{array}$ & $\begin{array}{l}\text { Muscle tone. Grade IIt: I: } 6.94 \\
\text { (2.06), C: } 7.48 \text { (2.20); P<0.05; grade } \\
\text { III: I: } 11.17 \text { (2.57), C: } 12.44 \text { (3.32); } \\
\text { P<0.01. Reflexes. Grade II: I: } 4.54 \\
\text { (1.53), C: } 5.47 \text { (1.96); P<0.01; grade } \\
\text { III: I: } 9.29 \text { (2.57), C: } 10.33 \text { (2.74); } \\
\text { P<0.01. Consciousness. Grade II: I: } \\
\text { 3.19 (1.04), C: } 3.88 \text { (1.49); P<0.05; } \\
\text { grade III: I: } 5.14 \text { (1.81), C } 6.44 \\
\text { (1.87); P<0.01 }\end{array}$ & NA; none & $\begin{array}{l}\text { I: } 16.2 \%(6 / 37), \text { C: } \\
26.5 \%(9 / 34) ; P<0.01\end{array}$ & NA & None \\
\hline Lu 199925* & $\begin{array}{l}\text { I: } 97 \%(31 / 32), \text { C: } 70 \%(21 / 30) ; P<0.05 \\
\text { (clinical symptoms disappeared in } 10 \text { days) }\end{array}$ & NA & $>10$ days; none & NA & NA & None \\
\hline Si $1999^{26}$ & $\begin{array}{l}\text { I: } 93 \% \text { (53/57), C: } 76 \%(37 / 49) ; P<0.01 \\
\text { (clinical symptoms and convulsions } \\
\text { disappeared in } 10 \text { days) }\end{array}$ & Days in hospital. I: 12.8, C: 17.6 & $\begin{array}{l}3 \text { months to } 4 \text { years; } \\
\text { none for total } \\
\text { effectiveness, not clear } \\
\text { for long term sequelae }\end{array}$ & $\begin{array}{l}\text { I: } 7.0 \%(4 / 57), \text { C: } 12.2 \% \\
(6 / 49)\end{array}$ & $\begin{array}{l}\text { I: } 5.3 \%(3 / 57) \\
\text { C: } 16.3 \%(8 / 49)\end{array}$ & NA \\
\hline Song $2000^{27 \star}$ & $\begin{array}{l}\text { I: } 90 \% \text { (46/51), C: } 66 \%(33 / 50) ; \mathrm{P}<0.01 \\
\text { (clinical symptoms disappeared in } 14 \text { days) }\end{array}$ & NA & 6-14 months; I: 3, C: 11 & $\begin{array}{l}\text { I: } 18.8 \%(9 / 50), C: \\
43.6 \%(17 / 50) ; P<0.05\end{array}$ & $\begin{array}{l}\text { I: } 5.9 \%(3 / 51) \\
\text { C: } 22.0 \% \\
(11 / 50)\end{array}$ & NA \\
\hline Sun $2000^{32}$ & $\begin{array}{l}\text { I: } 93 \% \text { (39/42), C: } 58 \% \text { (33/40); (cured, } \\
\text { improved, alive; time to outcomes not given) }\end{array}$ & NA & Not clear; none & NA & $\begin{array}{l}\text { I: } 7.1 \%(3 / 42) \\
\text { C: } 17.5 \%(7 / 40)\end{array}$ & NA \\
\hline Sun $1998^{33}$ & $\begin{array}{l}\text { I: } 100 \%, \text { C: } 76 \% \text { (numbers not given); } \\
\text { P }<0.01 \text { (measurement index not mentioned) }\end{array}$ & NA & $N A ; N A$ & NA & NA & None \\
\hline Wang $2002^{34}$ & NA & $\begin{array}{l}\text { Seizure controlled; recovery of } \\
\text { muscle tone, reflexes, and } \\
\text { consciousness. } 4-6 \text { days fewer in I } \\
\text { than C }\end{array}$ & NA; none & NA & NA & NA \\
\hline Wang $1999^{35}$ & $\begin{array}{l}\text { I: } 97 \% \text { (22/23), C: } 68 \% \text { (15/22); P<0.05 } \\
\text { (clinical symptoms disappeared in } 10 \text { days) }\end{array}$ & NA & $>10$ days; none & NA & NA & NA \\
\hline Wang $2001^{36}$ & $\begin{array}{l}\text { I: } 100 \%(80 / 80), \text { C: } 80 \% \text { (64/80); P<0.05 } \\
\text { (cerebral oedema—by computed } \\
\text { tomography—disappeared in } 10 \text { days) }\end{array}$ & $\begin{array}{l}\text { Symptoms. At least } 3 \text { days fewer in } \\
\text { I than C }\end{array}$ & 10 days; none & NA & NA & NA \\
\hline Wang $2001^{37}$ & $\begin{array}{l}\text { I: } 90 \%(76 / 84), \text { C: } 76 \%(61 / 80) ; P<0.05 \\
\text { (clinical symptoms disappeared in } 1 \text { week) }\end{array}$ & NA & Not clear; none & NA & NA & NA \\
\hline Wen $2001^{38}$ & NA & $\begin{array}{l}\text { Muscle tone. I: 9.13, C: } 11.80 \\
\text { consciousness: I: } 4.37, \text { C: } 5.60\end{array}$ & Not clear; NA & NA & NA & NA \\
\hline Yuan $1999^{39}$ & $\begin{array}{l}\text { I: } 93 \% \text { (28/30), C: } 70 \%(21 / 30) ; P<0.05 \\
\text { (clinical symptoms and cerebral oedema } \\
\text { disappeared in } 10 \text { days. How cerebral } \\
\text { oedema was detected not given) }\end{array}$ & NA & $>10$ days; none & NA & NA & NA \\
\hline Zhang $2000^{40}$ & NA & $\begin{array}{l}\text { Symptoms. I: } 5 \text { (2.9), C: } 7.8 \text { (1.2); } \\
\text { P<0.001 }\end{array}$ & Not clear; none & NA & NA & $\begin{array}{l}\text { Rentrolental } \\
\text { fibroplasia I: } \\
1.6 \%(1 / 60) \\
\text { C: } 1.8 \% \\
(1 / 56)\end{array}$ \\
\hline
\end{tabular}

$\mathrm{C}=$ control group; I=intervention group, NA=not available.

${ }^{*}$ Alternate patient allocation.

†Severity grade: I=mild, II=moderate, III=severe.

trials studied between 40 and 198 patients. Four different sets of criteria were used to diagnose hypoxic-ischaemic encephalopathy (see table 1). ${ }^{37-40}$ These sets are similar and the criteria used are an abnormal obstetric history of fetal anoxia and distress; asphyxia after birth resulting in a low Apgar score and disturbance of consciousness; change in muscle tone; and abnor- mal reflexes within 12 hours of birth. The severity of hypoxic-ischaemic encephalopathy varied and grading of severity was probably not applied uniformly across the trials. Trials used various doses of hyperbaric oxygen and some had additional treatments, such as antioxidants and neurotrophic agents in each arm. Populations and the delivery of hyperbaric 


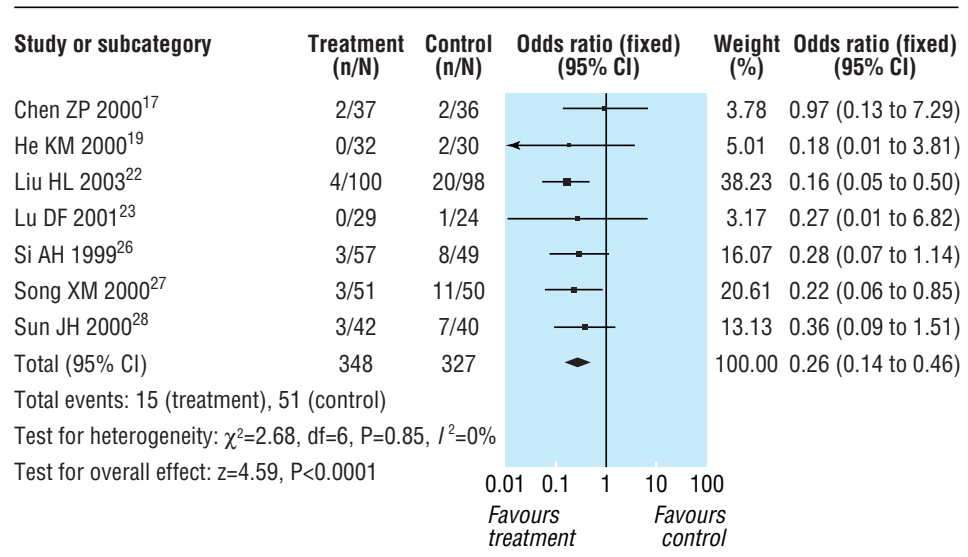

Fig 2 Effect of treatment with hyperbaric oxygen on mortality in hypoxic-ischaemic encephalopathy

oxygen varied greatly. Table 1 shows the characteristics of the studies.

Seventeen of the 20 studies mentioned "random" in the methods section, but few other trial details were given. None of the randomised trials mentioned the method of sequence allocation or whether allocation was concealed. In the other three studies treatment was allocated on an alternate basis. They were included because order of birth was considered to be a random event. $^{22} 2527$ Selection bias may have been more prominent in these studies, but because of the uniformly poor methodological quality of reporting this could not be determined. Only one trial mentioned blinding (of outcome assessment)..$^{22}$ No trials with losses to follow-up described reasons for the losses. The only trials with intention to treat analysis were those without losses to follow-up. Figure 1 shows quality factors.

Table 2 shows the results of the studies. Not all outcomes were reported in each trial, but overall, treatment with hyperbaric oxygen had a better outcome than the comparator. Only seven trials reported mortality (fig 2). Hyperbaric oxygen significantly reduced mortality in hypoxic-ischaemic encephalopathy (odds ratio $0.26,95 \%$ confidence interval 0.14 to 0.46 ). Seven trials measured neurological sequelae (fig 3). Neurological sequelae were significantly reduced in neonates treated with hyperbaric oxygen compared with controls $(0.41,0.27$ to 0.61$)$. Little heterogeneity was seen between the trials for both comparisons.

Adverse events were reported in only one trial-rentrolental fibroplasia occurred in one case each in the intervention group and the control group at follow-up. ${ }^{36}$ Seven trials reported no adverse events, and the remainder did not mention adverse events (table 2).

\section{Discussion}

The results of this systematic review suggest that treatment with hyperbaric oxygen may reduce mortality and neurological sequelae in term neonates with hypoxic-ischaemic encephalopathy. Hyperbaric oxygen has been used to treat various conditions for several decades and has been used in neonates. Although this form of treatment is controversial, it has developed rapidly in China over the past decade and is widely used there.

\section{Limitations}

Trial reports were of poor quality according to the criteria of the York Centre for Reviews and Dissemination, were not written to CONSORT standards, and lacked many details. Publication bias is a possibility as studies with negative results may not have been published. The 20 trials differed greatly in terms of the severity and status of the condition, exposure to hyperbaric oxygen, time to treatment and other baseline characteristics, and the measurement of outcomes. In addition, little information was given on side effects such as retrolental fibroplasia.

\section{Implications}

An adequately powered, high quality, randomised controlled trial is needed to investigate the effectiveness of hyperbaric oxygen in term neonates with hypoxic-ischaemic encephalopathy. If the effectiveness of this treatment is confirmed, this will have two

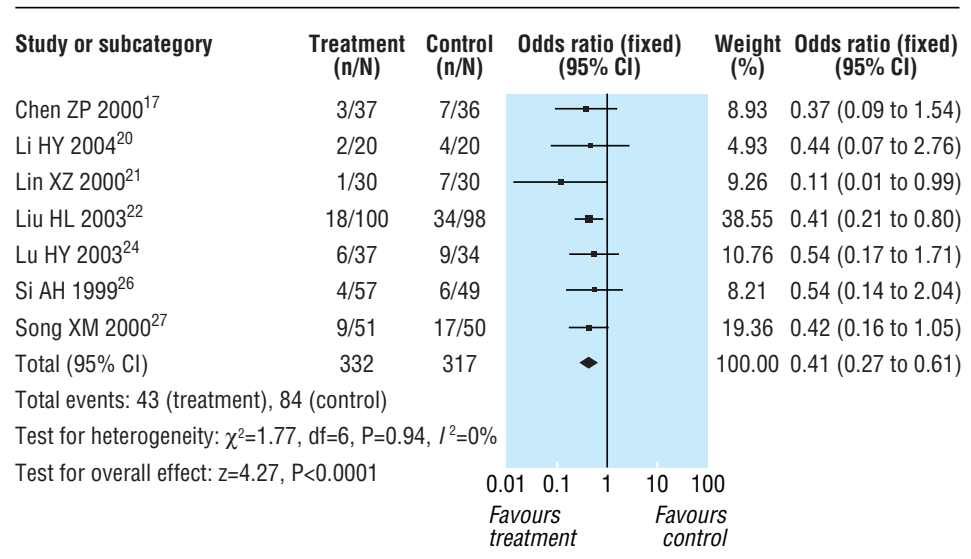

Fig 3 Effect of treatment with hyperbaric oxygen on neurological sequelae in hypoxic-ischaemic encephalopathy 


\section{Research}

main implications. Firstly, the treatment of hypoxic-ischaemic encephalopathy will change radically in the West and hyperbaric oxygen chambers will be required in all special care baby units. The costs of providing this treatment could be high, but they might be outweighed by fewer neonatal deaths and reduced requirements for specialist paediatric medical and nursing care. Secondly, evidence of the effectiveness of this treatment came from Chinese sources that are not routinely searched when systematic reviews are carried out in the West. It is not known at present how much useful evidence will be found once researchers start to look. This may also be true for evidence collected in Russia. To determine whether the inclusion of Chinese and Russian trials would reinforce or change the conclusions of systematic reviews, Chinese and Russian trials of interventions should be reviewed and the results compared with currently available systematic reviews. In future, it may become general policy to check these databases, so systematic review groups would need reviewers skilled in these languages who also have access to the relevant databases and journals.

We thank Yuhua Zhang, Department of Neurology, the Fourth Hospital of Guilin, China and Xiaochao Chen, Department of International Trade, Xiamen University, China who helped with the hand search of Chinese journals.

Contributors: ZL conducted the original systematic review submitted for the masters degree in full. TX checked the inclusions and duplicate data extraction. CM supervised the original systematic review, wrote the journal article from it, and is guarantor.

Funding: None.

Competing interests: None declared.

Ethical approval: Not required.

1 Carli G, Reiger I, Evans N. One-year neurodevelopmental outcome after moderate newborn hypoxic ischaemic encephalopathy.J Paediatr Child Health 2004;40:217-20.

2 Newborn Services Clinical Guidelines Hypoxic Ischaemic Encephalopathy. www.adhb.govt.nz/newborn/guidelines/neurology/NE.htm (accessed 16 Dec 2004).

3 Pfenninger J, Bachmann D, Bendicht Wagner P. Survivors with bad outcome after hypoxic-ischaemic encephalopathy: full-term neonates compare unfavourably with children. Swiss Med Wkly 2001;131:267-72

4 Jacobs S, Hunt R, Tarnow-Mordi W, Inder T, Davis P. Cooling for newborns with hypoxic-ischaemic encephalopathy. Cochrane Database Syst Reu 2003;4:CD003311

5 Kent A, Kecskes Z. Magnesium sulfate for term infants following perinatal asphyxia (protocol). Cochrane Database Syst Rev 2003;2:CD004494.

6 Whitelaw A. Systematic review of therapy after hypoxic-ischaemic brain injury in the perinatal period. Semin Neonatol 2000;5:33-40.

7 Wei X, Zhao L, Ma Z, Holtzman DM, Yan C, Dodel RC, et al. Caffeic acid phenethyl ester prevents neonatal hypoxic-ischaemic brain injury. Brain 2004;127:2629-35.

\section{What is already known on this topic}

Hypoxic-ischaemic encephalopathy is a severe complication of asphyxia before, during, or after birth and occurs in 3.5-6/1000 live births

Current treatment in the West consists mainly of best supportive care

Hyperbaric oxygen is commonly used in China to treat this condition

\section{What this study adds}

This systematic review of 20 Chinese trials found that treatment with hyperbaric oxygen reduced mortality and neurological sequelae such as epilepsy, mental retardation, and cerebral palsy, but in all trials reporting of methods was poor and publication bias is a possibility

A high quality randomised controlled trial is needed to investigate and confirm the effectiveness of this treatment
8 Medical Services Advisory Committee. Hyperbaric oxygen therapy. Canberra: MSAC, 2001

9 Hailey D. Hyperbaric oxygen therapy-recent findings on evidence for its effectiveness. Update. Edmonton: Alberta Heritage Foundation for Medical Research, 2003

10 Guo S, Counte MA, Romeis JC. Hyperbaric oxygen technology: an overview of its applications, efficacy and cost-effectiveness. Int J Technol Assess Health Care 2003;19:339-46.

11 Aksenov I, Tonkopi F. Hyperbaric oxygen therapy in Russia-more than 60 indications: literature review. Undersea and Hyperbaric Medical Society Annual Scientific Meeting. Sidney, Australis, 25-29 May 2004. www (accessed 3 Mar 2006).

12 Richmond Hyperbaric Health Center. Summary of international indications for hyperbaric oxygen therapy. www.Richmond-hyperbaric.com/indications.html (accessed 1 Dec 2004)

13 Ramaswami RA, Lo WK. Use of hyperbaric oxygen therapy in Hong Kong. Hong Kong Med J 2000;6:108-12.

14 Chen ZL. Hyperbaric oxygenation for neonatal hypoxic-ischaemic encephalopathy. Chin J Practical Pediatr 1995;2:86-7.

15 Khan KS, ter Riet G, Glanville J, Sowden AJ, Kleijnen J, eds. Undertaking systematic reviews of research on effectiveness. York: NHS Centre for Reviews and Dissemination, 2001. CRD Report 4, 2nd ed. www.york.ac.uk/inst/crd/crdrep.htm (accessed 3 Mar 2006).

16 Deeks JJ, Altman DG, Bradburn MJ. Statistical methods for examining heterogeneity and combining results from several studies in meta-analysis. In: Eggar M, Davey Smith G, Altman DG, eds. Systematic reviews in health care, meta-analysis in context. London: BMJ Books, 2001:285-312

17 Chen ZP. Observation of the efficacy of hyperbaric oxygen in treating moderate and severe neonatal hypoxic-ischemic encephalopathy (in Chinese). Hua Xia Med 2000;2:155-6.

18 Ding YY, Chen XG, Chui N, Mei CM, Chen JQ, Wan XL, et al. Clinical study of hyperbaric oxygen in treating neonatal hypoxic-ischemic encephalopathy (in Chinese). Chin J Mod Clin Med 2003;1. Chinese Medical Journal (CMJ) Network. www.shouxi.net/ journal/200408/2125 25930.aspx (accessed 14 Oct 2004)

19 He KM. Effect of hyperbaric oxygen on neonatal hypoxic-ischemic encephalopathy (32 cases) (in Chinese). Chin J Contemp Pediatr 2000;2:345-6.

$20 \mathrm{Li} \mathrm{HY}$, Ding YX, Wang XN. Observation of the effect of hyperbaric oxygen in treating neonatal hypoxic-ischemic encephalopathy. Chin J Modern Chinese Medicine, 2(2B). July 2004 (in Chinese). Chinese Medical Journal Network. www.shouxi.net/journal/ 200407/209_8409.aspx (accessed 14 Oct 2004).

$21 \mathrm{Lin}$ XZ, Fu SY, Zhang AA, Liu XY. Evaluation of the efficacy of hyperbaric oxygen in treating hypoxic-ischemic encephalopathy of newborns according to neonatal behavioral neurological assessment scale (in Chinese). J Neonatol 2000;15:172-3.

22 Liu HL, Li LQ. Effect observation on treating newborn encephalopathy of hypoxic-ischemic (in Chinese). Chin J Pract Chin Mod Med 2003;3:770-1.

$23 \mathrm{Lu}$ DF. Observation of the efficacy of hyperbaric oxygen therapy in treating newborn with hypoxic-ischemic encephalopathy (in Chinese).J Chin Physician 2001;29:38.

$24 \mathrm{Lu}$ HY, Mao KX. Hyperbaric oxygen therapy in treating newborns with moderate and severe hypoxic-ischemic encephalopathy in 32 cases (in Chinese).J Xianning College (Med Sci) 2003;17:115-6.

$25 \mathrm{Lu} \mathrm{JF}$. Analysis of hyperbaric oxygen in treating neonatal hypoxic-ischemic encephalopathy in 32 cases (in Chinese). J Guangxi Med Univ 1999;16:703.

$26 \mathrm{Si} \mathrm{AH}$. Analysis of the prognosis of hyperbaric oxygen in treating neonatal hypoxic-ischemic encephalopathy (in Chinese). J Hyperbaric Oxygenation Med 1999-8. Beijing hyperbaric oxygen medicine research centre. www.chinahbo.org.cn/books/ gayyxzz/no14/40.htm (accessed 20 Sep 2004)

27 Song XM, Li X, Li JH, Wang SJ, Ding XC, Zhang ZX, et al. Observation of the efficacy of hyperbaric oxygen in treating neonatal hypoxic-ischemic encephalopathy (in Chinese). Chin J Practical Pediatr 2000;15:762.

28 Sun JH, Sun GY, Huang M. Complex treatment for neonatal hypoxic-ischemic encephalopathy in 42 cases (in Chinese). J Applied Clin Pediatr 2000;15:266.

29 Sun XJ, Sun SZ. Clinical observation of hyperbaric oxygen in treating neonatal hypoxic-ischemic encephalopathy in 32 cases (in Chinese). Chin J Birth Health Hered $1998 ; 6: 79$.

30 Wang AH, Li CX. Analysis of the curative effect of hyperbaric oxygen therapy in treating neonatal hypoxic-ischemic encephalopathy (in Chinese). Chin J Primary Med Pharm 2002;9:503.

31 Wang MM, Jiang SH, Yang XM. Report of hyperbaric oxygen in treating neonatal hypoxic-ischemic encephalopathy in 23 cases (in Chinese). Ningxia Med J 1999;21:113-4.

32 Wang SL, Yang L. Observation of the efficacy of hyperbaric oxygen therapy in treating newborns with hypoxic-ischemic encephalopathy (in Chinese). Shangdong Med J 2001;41:38.

33 Wang XJ, Liu JJ, Yun L, Wang XM, Lang ZY, Pang ZZ. Short-term efficacy and the effect on prognosis of hyperbaric oxygen treatment for neonatal hypoxic-ischemic encephalopathy (in Chinese). Chin J Contemp Pediatr 2001;3:273-4.

34 Wen R, Sha YJ, Wang MY, Yang SW, Xia Y. The effect of hyperbaric oxygen therapy on brain blood flow rate in treating neonatal hypoxic-ischemic encephalopathy (in brain blood flow rate in treating neonatal hypoxic

35 Yuan XW. Clinical observation of hyperbaric oxygen in treating newborns with hypoxic-ischemic encephalopathy (in Chinese). Mod Diagn Treat 1999;10:174

36 Zhang XH, Wang WQ, Chai S, Si XN, Luo ZY. Probe into efficacy and side effect of hyperbaric oxygen in treating neonatal hypoxic-ischemic encephalopathy (in Chinese). Chin J Birth Health Hered 2000;8:35-91

37 Han YK, for the Neonatology Group of Chinese Medical Association. Clinical diagnostic criteria and staging of neonatal HIE. Chin J Pediatr 1990;28:31.

38 Han YK, for the Neonatology Group of Chinese Medical Association. Diagnostic criteria and clinical grading of neonatal HIE. Chin J Pediatr 1997;2:99.

39 Hu YM, Jiang ZF. Practical paediatrics. 7th ed. Beijing: People's Medical Publishing House, 1995.

40 Jin HZ, Huang DM, Guan XJ. Practical neonatology. 2nd ed. Beijing: People's Medical Publishing House, 1997.

(Accepted 27 September 2005) 
Research

doi $10.1136 / \mathrm{bmj} 38776.731655 .2 \mathrm{~F}$

Department of Public Health and Epidemiology, University of Birmingham, Birmingham B15 2TT

Zulian Liu systematic reviewer
Tengbin Xiong PhD student

Catherine Meads director of masters degree in health technology assessment

Correspondence to: C Meads c.a.meads@bham.ac.uk

$\underset{3}{\infty}$ 